\title{
Aneurysmal Subarachnoid Hemorrhage: an Overview of Inflammation-Induced Cellular Changes
}

\author{
A.P. Coulibaly ${ }^{1} \cdot$ J.J. Provencio ${ }^{1,2}$ (i) \\ Published online: 6 January 2020 \\ (C) The American Society for Experimental NeuroTherapeutics, Inc. 2020
}

\begin{abstract}
Aneurysmal subarachnoid hemorrhage (SAH) is a devastating disease that leads to poor neurological outcomes and is characterized by both vascular and neural pathologies. Recent evidence demonstrates that inflammation mediates many of the vascular and neural changes observed after SAH. Although most studies focus on inflammatory mediators such as cytokines, the ultimate effectors of inflammation in SAH are parenchymal brain and peripheral immune cells. As such, the present review will summarize our current understanding of the cellular changes of both CNS parenchymal and peripheral immune cells after SAH.
\end{abstract}

Keywords Subarachnoid hemorrhage $\cdot$ Inflammation $\cdot$ Microglia $\cdot$ Astrocytes $\cdot$ Neutrophils $\cdot$ Delayed cerebral injury

\section{Introduction}

Aneurysmal subarachnoid hemorrhage (SAH), the collection of blood in the subarachnoid space after an aneurysm rupture, accounts for 5-10\% of all stroke patients in the USA [49]. With a varied demographic, SAH is more prevalent in the population 50 years and older [49]. Thirty to $50 \%$ of SAH survivors develop delayed neurological dysfunction [30] leading to decreased quality of life and increased societal monetary burden $[9,51]$. This delayed neurological dysfunction (DND) is poorly understood but is thought to be, in part, caused by secondary brain injury due to delayed cerebral ischemia (DCI). Recent evidence demonstrates that both ischemic and non-ischemic brain parenchymal damage contribute to the development of DND [34].

A defining characteristic of SAH is the development of spasm in large cerebral vessels. Vasospasm has been documented at both early and chronic periods of the injury [1, 65]. A leading hypothesis in DCI assumes that cerebral vasospasm causes global ischemic tone in the SAH brain. This, in turn, leads to neuronal dysfunction and, ultimately, cognitive

J.J. Provencio jp3b@virginia.edu

1 Department of Neurology, University of Virginia, Charlottesville, VA, USA

2 Department of Neuroscience, University of Virginia, Charlottesville, VA, USA deficits. As such, preclinical and clinical trials of vasodilators were undertaken. Unfortunately, most of these studies clearly show that the resolution of cerebral vasospasm does not attenuate the development of DND after SAH. For example, the administration of the endothelin-1a antagonist clazosentan ameliorates vascular spasm without improving outcomes [36]. On the other hand, administration of nimodipine, a calcium-channel blocker, attenuates the neurological outcome with no change in vascular spasm $[30,50]$. These results demonstrate that, though important, cerebral vasospasm is, at best, not the only cause of DND. Re-evaluation of older data and new studies have led to a new hypothesis that immune system activation (i.e., inflammation) in the CNS is critical to the development of DND [34].

Cytokine analysis has shed some light on the possible mechanisms involved in DND (there are currently excellent reviews detailing the inflammatory cytokine profiles in the brain and periphery after SAH [34] and their contribution to vascular dysregulation [55]. Indeed, many clinical trials targeting inflammatory molecules have been undertaken (reviewed in [14]). However, the ultimate effectors of damage are inflammatory cells. To date, many studies have focused on the contribution of inflammatory cells to vascular spasm after SAH (Reviewed by [5] with very little focus to their direct effect on brain cell function. The study of inflammation's direct contribution to SAH-induced DND is a nascent field; therefore, little is known about the cellular changes that occurs in both parenchymal and non-parenchymal cells, including inflammatory cells, after SAH. As such, we will take the 
approach of defining the state of non-neuronal parenchymal cells and the recruitment of innate immune cells into the CNS after SAH that lead to brain damage.

\section{SAH Temporal Pathophysiology}

Most studies of SAH, including ours, focus on either understating the immediate effect of the hemorrhage on cellular function or its delayed effect on cognition. In the human population, the early aspect of the injury, acute phase or early brain injury, accounts for the first several hours, $0-72 \mathrm{~h}$, after the hemorrhage [58]. As previously described, cognitive deficits develop in the clinical settings between 6 and 14 days after hemorrhage [60]. In preclinical studies, the early brain injury or acute phase seems restricted to the first $24 \mathrm{~h}$ after the hemorrhage [57], while cognitive deficits, a result of DND, develops 6-8 days after SAH [44]. In light of these data, we propose that these symptomatic periods point to 3 specific phases of injury in the CNS after SAH. An acute, or early brain injury, phase is the first $24 \mathrm{~h}$ after injury in rodents. A sub-acute phase includes the period between the acute phase and the onset of DND. We postulate this period to be between 1 and 3 days after injury. Of note, 3 days is used as an upper limit because our research has shown that the peak of neutrophils infiltration in the rodent CNS after SAH occurs at day 3. Finally, the chronic phase of the injury includes the development of delayed neurological dysfunction. For the remainder of this review, we will focus on the preclinical data available in the literature and organize the cellular and molecular changes using the above temporal organization (acute, sub-acute, and chronic phases).

\section{Acute Phase or Early Brain Injury (0-24 h Post Hemorrhage)}

This is a period that is commonly referred to as early brain injury (EBI) after SAH. As early as 10 min after hemorrhage, neurodegeneration and cell death are observed in the brain [13]. Dying cells are seen in close proximity to areas of bleeding, i.e., basal cisterns. Indeed, Friedrich and colleagues show that $10 \mathrm{~min}$ after SAH, TUNEL-, and Fluorojade-positive cells are observed in the brain [13]. The number of TUNEL positive cells peaks in the brain at $24 \mathrm{~h}$ in the somatosensory cortex, hippocampus, striatum, and basal cortex [4, 13, 43, 46]. Death markers, including cleaved caspase-3, are widespread and colocalize with neurons, astrocytes, and endothelial cells $[13,18,46]$. Although the above studies focused on apoptotic cell death, evidence of other cell death mechanisms, including autophagy, necrosis, and necroptosis, have been documented in the acute phase of $\mathrm{SAH}$, i.e., between 0 and $24 \mathrm{~h}[13,61,63,70]$. Though yet unknown, it is likely that this early neuronal loss may be due to a change in the metabolic state of the brain from both ischemia and breakdown of the blood-brain barrier. Recent reports have demonstrated that the intercellular transfer of mitochondria is critical to the survival of stressed/damaged cells (reviewed by [32]). We further propose that this metabolic dysfunction contributes to the rise of inflammation-inducing types of cell death like necrosis and necroptosis. It is unknown (and of interest) whether this early loss of neurons coincides with the onset of mitochondria dysfunction. Furthermore, in this period, injured neurons and dying cells release inflammatory molecules, such as the danger signal high-mobility group box 1 (HMGB1), in the extra cellular space [61]. These danger signals act as triggers to initiate or drive inflammation in the CNS [34].

At the time when cell death is observed in the brain, increased breakdown in the blood-brain barrier, cortical edema, and the presence of acute spasm (different than delayed cerebral vasospasm) in the basilar and middle cerebral arteries are also apparent $[4,44]$. Although acute spasm occurs in this period, it is mainly reported in larger vessels [25] at the base of the brain. Later in the delayed period, vascular spasm has been associated with SAH pathology. There is little evidence of early vascular spasm as a driver of poor outcome. More studies are needed to truly understand the role of early spasms in the etiology of SAH-induced DND.

The hemorrhage also leads to increased intracranial pressure and decreased cerebral blood flow (even brain circulatory arrest in some cases). The decrease blood flow causes a transient energy imbalance in the brain with metabolically active cells deprived of substrate [10]. This energy imbalance is thought to contribute to the switch from apoptotic cell death within the brain to a necrotic cell death [13]. In addition, the energy imbalance leads to an ionic imbalance within neurons and astrocytes thereby creating conditions that promote the generation of cortical spreading depression (CSD). Indeed, a recent study demonstrated the infusion of blood in the subarachnoid space of the swine brain can lead to repetitive CSD in the first $6 \mathrm{~h}$ [22]. Furthermore, recent evidence demonstrated that the presence of CSD exacerbates brain injury after SAH [20]. These data suggest that although the direct contribution of CSD to DND is unclear, it most likely contributes to the overall injured state of the brain. In all, it is clear that EBI is associated with worse outcome and may set the stage for later damage; however, it is less clear how important it is for mediating long-term disability.

\section{Sub-acute Phase (1-3 Days Post Hemorrhage)}

As stated earlier, most studies of SAH focus on changes in the acute/EBI phase or the delayed neurological dysfunction, DND, phase after the hemorrhage. As such, there is less evidence addressing the cellular and molecular changes in animal models of SAH in the "sub-acute" period after the hemorrhage. Here we summarize those findings and make the argument that more studies need to be undertaken to get a better understanding of CNS changes leading to DND. 
The pathological state of the brain becomes more pronounced as the blood within the subarachnoid space degrades into products that further aggravate the environment within and around the brain. Blood breakdown products not only affect the vasculature adversely, but are also cytotoxic to parenchymal cells $[35,38,52,57]$. As such, we would expect that as blood degradation continues, more deleterious effect will be observed in the parenchyma. Indeed, more neuronal and glia loss is present in this period [46]. Unlike the acute period, the cell death observed here is not apoptotic [46], suggesting a possible metabolic deficits or the presence of cell toxic elements. This additional loss of cells may set the stage for the later development of DND associated with SAH.

The toxic environment after SAH also affects early recovery. A recent study, characterizing the effect of SAH on the brain neurogenic zones, shows a significant decrease in cell proliferation within the subventricular and subgranular zones of the brain between days 1 and 3 after SAH [31, 72], suggesting that these cells are also vulnerable to the toxic/hypoxic brain milieu after SAH. The proliferative rate however starts recovering after day 3 , suggesting that the CNS milieu becomes less deleterious after this period. These data suggest that the activity of immune cells, parenchymal and peripheral, and other scavenger cells during this period maybe critical to the overall state of the brain in the later phase of SAH. It is possible that determining the molecular changes in this period may well distinguish those who will later develop DND and those who will not. Finally, it is also likely that a decrease in regenerative potential of the brain, starting in the sub-acute period, adversely affects the potential for neurological recovery over time.

\section{Delayed and Chronic Injury Phase (Delayed: 3-14 Days Post Hemorrhage; Chronic: > 14 Days)}

In both human and animal models, the delayed injury period of SAH is when many cognitive deficits arise. In animal studies, deficits in spatial learning are evident in this period [44]. During this delayed period, many of the physiological changes observed in the acute phase of injury, edema, cerebral blood flood, intercranial pressure, etc. [4] subside. The blood clot present in the subarachnoid space has begun to visibly degrade. Vasospasm reoccurs (Delayed cerebral vasospasm) during this period but takes on a different morphology with vascular wall thickening in addition to muscle spasm $[4,44]$. A recent study demonstrates a second wave of neuronal death in the brain from days 14 to 28 after the hemorrhage [56], suggesting that the events around this period lead to further and important brain damage. This period takes on added importance because the delay between the injury and delayed worsening may be an opportunity for therapeutic intervention.
The chronic phase is when the acute and sub-acute injuries have passed, and the brain is beginning to recuperate. It is likely that this phase overlaps significantly with the delayed injury phase. This period is denoted by an increase in neurogenesis in both the subventricular and subgranular zones [31, 72]. Newly divided cells express mature neuronal markers and are detected in both the striatum and granule cell layer of the hippocampus suggesting an integration into the neuronal circuitry [72]. High proliferation rates are also observed in the astrocytic population of the brain. The increased proliferation is a sign of brain attempting to heal itself. This period may present another window at which interventions can help maximize the effect of intrinsic brain repair processes.

\section{Cellular Actors in Inflammation After SAH}

Studies of cytokines and other immune proteins are difficult to interpret because their effects are often context specific and outcomes vary depending on how these mediators interact with each other. Ultimately, inflammatory effects on the brain are mediated by the cells that act as effectors. To develop a complete enough understanding of this system and develop rational therapies will require a better understanding of the cellular changes that occur during SAH. Therefore, for the rest of this review, we will focus on providing a clear temporal characterization of immune and parenchymal cells shown to be critical to the brain injury associated with SAH (Fig. 1).

The inflammatory system of the brain is different than that of other tissues in a number of noteworthy ways. The tissue resident inflammatory cells of the brain, the microglia, come from a different origin than the monocyte/macrophage/dendritic cells that make up the tissue resident inflammatory cells outside the brain. The other major glial cell types, astrocytes, and oligodendrocytes (in the form of oligodendrocyte precursor cells or OPCs) also have roles in inflammation in the brain. In addition, peripheral inflammatory cells such as monocytes and neutrophils are clearly important actors in the development of brain pathology but are often restricted in how they enter the CNS. In this next section, we will discuss these cell types and how they impact outcomes after SAH.

\section{Resident Brain Cells}

\section{Microglia}

Microglia are the resident immune cells of the CNS. Unfortunately, there are few recognized markers of microglial function other than morphological changes associated with activation (which may or may not indicate a specific inflammatory function). Therefore, there remains significant controversy about the role microglia play in mediating EBI, DCI, and brain damage after SAH. Recent studies have investigated 
Fig. 1 Temporal changes in cellular elements associated with SAH. Neutrophil accumulation, microglial activation, and neuronal cell loss appear to have two peaks (acute phase and delayed injury phase), whereas monocytes and macrophages appear to increase from the subacute phase to the recovery phase suggesting that they are important in recuperation and remodeling of the brain after injury

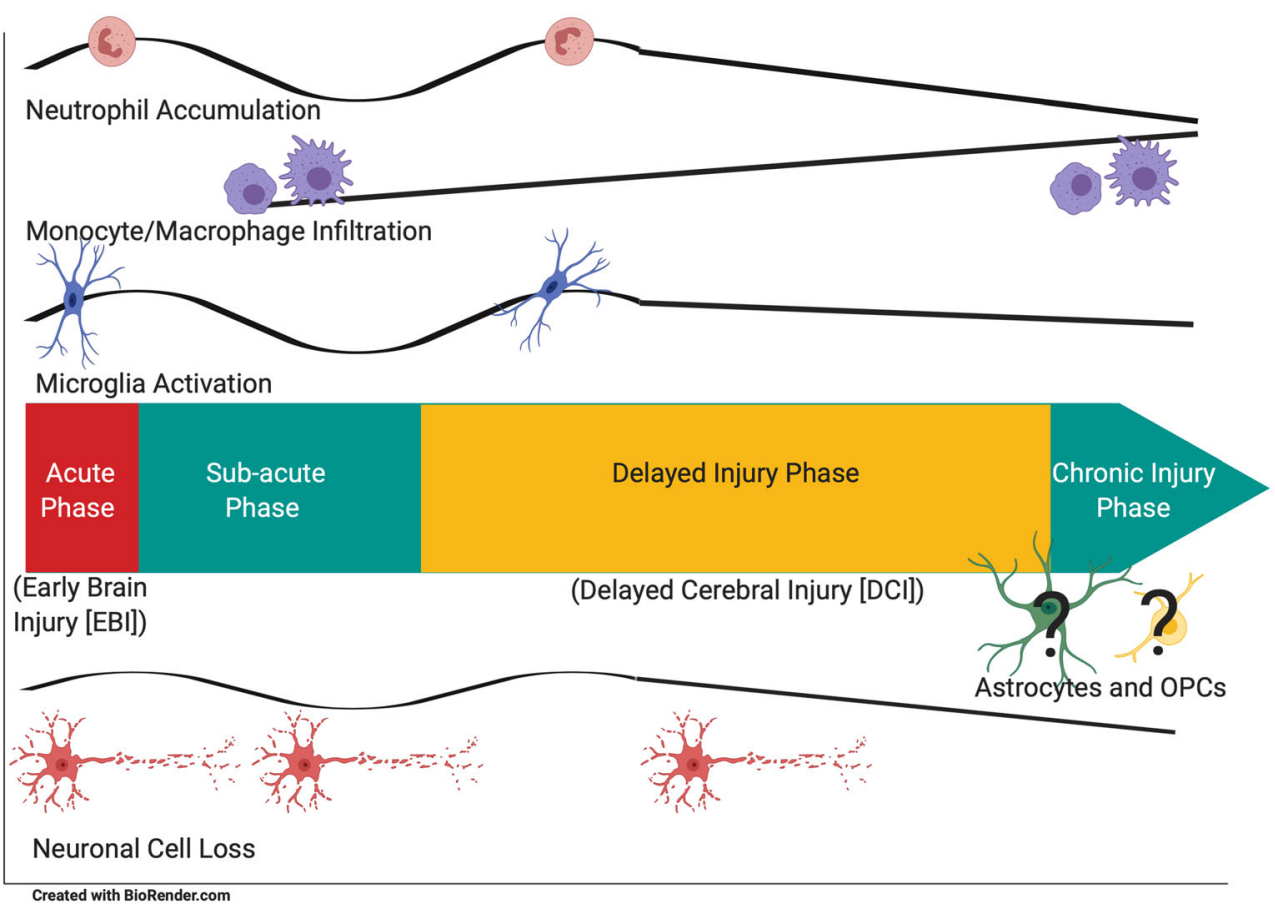

brain injury after manipulation of microglia which begin to shed light on the role of microglia in SAH. The loss of functional heme-oxygenase-1 (a potent cytoprotective enzyme in cellular stress) leads to increased neuronal death, increased vasospasm, and worsened cognitive deficits after SAH suggesting that microglia may play a protective role again radical stress [54]. However, microglial depletion leads to decreased neuronal loss and improved vasospasm after SAH suggesting conversely that microglia may worsen cell injury [2]. The main difference between these two studies is the timing of the depletion/inactivation of microglia. Schallner et al. [54] focused on the microglia activation at the 24-h time point, while the Atangana et al. [2] study focused at the 7- and 15day time points. It has recently been suggested that the timing of activation of microglia, in acute vs chronic injury phase, can dictate the inflammatory versus recuperative status of these cells after injury [41]. Therefore, understanding temporal changes in activation following SAH is important to our overall understanding of SAH etiology.

\section{Acute Injury Period}

Within $24 \mathrm{~h}$ of the hthe basal cistern, and leads to as yetemorrhagic event, microglia show morphological signs of activation $[18,44]$. The activation is more prevalent in brain regions adjacent to the location of the blood clot [44], i.e., the basal cistern, and leads to as yet unidentified processes that promote cell death, including necroptosis in neurons [70]. Neuronal necroptosis and necrosis leads to further release of inflammatory molecules (alarmins) such as HMGB1 [61], which further activate microglia. Their activation state during this acute period is thought to be proinflammatory [64], and leads to the recruitment of blood-derived innate inflammatory cells, permeability of the blood-brain barrier and increased neuronal loss. Although there is evidence in development that microglia can be phagocytic (particularly in the pruning of synapses), there is no evidence that they perform this function in the acute period after SAH.

The exact mechanism by which microglia lead to cell death after SAH is not yet understood. One possible mechanism has been investigated in ischemic stroke research. In the middle cerebral occlusion model of stroke, a spreading calcium wave, a CSD phenomenon, develops specifically in the microglia population [62]. As mentioned above, in the acute phase of SAH, ionic imbalance leads to CSD in the cortex $[22,53]$. Whether CSD contributes to the activity of microglia is still unknown. Because calcium is critical for the activation of immune cells [17], spreading depolarization waves in microglia may be a mechanism by which microglia response to the injury may be affected early after the hemorrhage. More studies will need to be conducted to determine the effect of CSD on microglia during this phase of the injury.

\section{Sub-acute Injury Period}

To date, little is known about the state of microglia between the acute period and the delayed period following SAH. Our own research has focused on the activation of microglia at $24 \mathrm{~h}, 3$ and 6 days after SAH [44]. There is little evidence to the molecular or cellular changes observed in microglia in this period. Histological changes observed in microglia after 3 days (summarized below) indicate a dynamic change to these cells 
between their initial activation at $24 \mathrm{~h}$, return to baseline 3 days, and reactivation at 14 days after SAH. As such, this limited understanding of the cellular mechanisms associated with the activation phenotype and their implications on neuronal injury or recruitment of other inflammatory cells greatly hinders our understanding of the role these cells play in SAH. Our laboratory is interested in better understanding the role of microglia during the in-between period between EBI and the onset of DND.

\section{Delayed and Chronic Injury Period}

Between days 4 and 6 microglia are less activated morphologically than what is observed at $24 \mathrm{~h}$ but greater than at baseline $[44,56]$. The activation increases, peaks a second time around day 14 , and subsides by day 28 $[29,56]$. Between days 14-28, microglia increase their expression of interleukin-6, toll like receptor 4 , and tumor necrosis factor alpha (TNF $\alpha)[21,56]$; immune modulators shown to open the blood-brain barrier and recruit peripheral immune cells [26]. Attenuation of this latter microglia activation and/or depletion decreases neuronal cell death suggesting that this time period remains active immunologically and does not reflect complete recovery [2].

Many questions remain unanswered regarding the role microglia play, phagocytic, inflammatory, neurogenic, etc., in both EBI, DCI, and DND after SAH. Microglia play both neuroprotective and neuroinflammatory roles under different injury contexts. Furthermore, there are other roles of microglia that have not yet been investigated. For example, a recent report demonstrates that microglia interact with the microvasculature to regulate energy metabolism in the brain [71]. Although the role of microglia as the predominant inflammatory cell in the brain has been called into question, to understand how inflammation leads to DND and brain damage, it will be important to have a deeper understanding of how microglia interact with other inflammatory and noninflammatory cells after SAH.

Finally, it is worth mentioning that the inability to truly distinguish between microglia, perivascular macrophages, and infiltrating monocytes has made it difficult to determine whether the changes attributed to microglia after SAH are not from other immune cells. The advent of deep sequencing tools, like single cell RNA sequencing, has led to the identification of markers that are unique to the microglia population, i.e., P2ry 12 and Tmem19 [19]. More studies using these newly identified markers would greatly enhance our understanding of the role microglia play in SAH etiology.

\section{Astrocytes}

Astrocytes play an important role in the homeostasis of the CNS. They have varied roles including supporting neuronal electrical potentials, improving transsynaptic communication, structurally supporting neurons, and regulating some forms of inflammation in brain. In addition, astrocytic endfeet make up a major component of the blood-brain barrier and their interaction with the vasculature plays a critical role in the movement of molecules, including glucose, into the brain parenchyma and in regulating blood flow [37]. To date, little is known regarding the effect of astrocytes on the pathology of SAH. This is an area that is ripe for study that may lead to important insights and possibly treatments.

Recent reports show that CSD (which are observed after $\mathrm{SAH}[22,53])$ influence the activity of astrocytes. CSD leads to increased production of proinflammatory molecules, glial fibrillary acid protein, and vimentin in astrocytes both in vivo and in vitro [15]. In response to CSD, astrocytes upregulate their expression of the inflammatory receptor toll like receptor 3. The activation of this receptor, using poly I:C, has proven beneficial in a middle cerebral artery occlusion model of acute ischemic stroke and in mitigating the effect of CSD on neuronal damage [16, 42]. This suggests that astrocytes regulate the inflammatory state of the brain in response to injury. As such, it is reasonable to conclude that astrocytes contribute to the inflammation observed in the brain after SAH. Because so little work has been done in this area, it is difficult to make any firm conclusions about what role astrocyte-driven inflammation has in damage after SAH.

\section{Oligodendrocyte Precursor Cells}

Oligodendrocyte precursor cells (OPCs) were once thought to function only as progenitors of oligodendrocytes. In fact, it is now accepted that a certain percentage of OPCs never "mature" into oligodendrocytes and have roles that are completely different than myelination. This is evidenced in models of multiple sclerosis where OPCs are attracted to areas of inflammation but often do not differentiate into oligodendrocytes. The role of these cells in inflammatory areas is still poorly understood. It is possible that OPCs may act as support for the "injured" neurons in inflamed regions of the brain after $\mathrm{SAH}$. Indeed, OPCs have been shown to release factors that promote neuronal survival [66]. OPCs respond to both demyelinating and non-demyelinating injuries to the brain reinforcing the hypothesis that, like other parenchymal cells, they are vulnerable to inflammatory milieu of the brain [11]. To date, very little is known of the effect of SAH on these cells. It is possible that since SAH leads to brain inflammation, OPC activity changes both in response to blood product in the acute phase and inflammation in the chronic phase after SAH.

A recent hypothesis proposes that cerebral edema can cause demyelination in certain regions of the brain [3]. The authors postulate that the increased pressure, leading to mechanical stress, and oxygen and glucose deprivation, can contribute to loss of myelin and parenchymal cells. This suggests 
that brain edema is an early phenomenon after SAH may lead to activation of OPCs. Current evidence suggests that OPCs are a source of matrix metalloproteinase 9 (MMP9). MMP9 is implicated in the breakdown of the vascular basement membrane, which promotes peripheral cell recruitment into the CNS [59]. Although no specific study of OPC derived MMP9 has been undertaken in SAH to our knowledge, it is likely that the activation of OPCs may contribute to the recruitment of peripheral immune cells through the breakdown of the blood-brain barrier. Overall, it is too early to speculate on the role of OPCs in SAH.

\section{Systemic Inflammatory Cells}

There is little evidence that cells of the adaptive immune system, i.e., lymphocytes, play a significant role in SAH. This is likely the combination of the limited role they play and limited research on these cells in the SAH field. As such, we will focus on the innate immune system, where the data is more robust.

\section{Monocytes/Macrophages}

Monocytes are innate immune cells produced predominantly in the bone marrow, and released into the blood stream that enter tissue for both surveillance and inflammation. Very rapidly after entering a tissue, including the brain, monocytes differentiate into macrophages and take on a different function. Very little is known of the monocytic contribution to SAH etiology. This is in part due to the limited resources available to distinguish infiltrating monocytes from microglia and perivascular macrophages in the central nervous system. Therefore, intraparenchymal monocytes are often counted as microglia, since the same markers identify both populations. Furthermore, once monocytes infiltrate brain tissue, they mature into macrophages and present a different morphological and biochemical profile. To date, there are limited studies specifically looking at monocytes after SAH. The work that has been done has focused on their role in the acute and subacute period after the injury. Jedrzejowska-Szypulka and colleagues show a high number of infiltrating monocytes in the brain $24 \mathrm{~h}$ after SAH [24]. In addition, 1 and 2 days after SAH, an increase in Ly6 $\mathrm{C}^{\text {hi }}$ monocytes is observed in the brain parenchyma suggesting a continuous infiltration of these cells during these periods [18]. Furthermore, Xu and colleagues use a currently available transgenic tool to parse out the infiltration of blood derived monocytes in the brain after SAH [68]. Using the markers $\mathrm{CxCr} 1$ (microglia) and CCR2 (monocytes), the authors were able to clearly demonstrate that monocytes enter the brain parenchyma no earlier than 3 days after SAH, which raises the question of whether what has been previously described as microglial activation observed in the chronic phase of the injury is possibly, in part, due to infiltration of monocytes and macrophages?

It is likely that monocytes and macrophages play a role in causing inflammation or recruiting inflammatory responses after SAH. Newer tools that allow the discrimination of monocyte/macrophages from microglia will aid in our understanding of their role in $\mathrm{SAH}$.

\section{Neutrophils}

Neutrophils are the most abundant type of leukocytes found in the circulation. These granulocytes have been implicated in tissue damage in both infectious and sterile injury. Many studies have demonstrated neutrophil infiltration after SAH. Our laboratory has shown that early depletion/removal of neutrophils from the peripheral circulation abolishes DND after SAH [44], demonstrating that these cells are critical to the secondary injury leading to DND. Furthermore, we demonstrated that neutrophils, through yet unknown mechanisms, modulate the expression of NMDAR subunits in the hippocampus leading to spatial memory deficits [45]. Unfortunately, our understanding of the importance of neutrophils after SAH is limited by gaps in the available data regarding their temporal accumulation and the mechanisms of action within the CNS after SAH.

\section{Acute Injury Period}

The hallmark inflammatory response in SAH is the infiltration of the CNS by peripherally derived immune cells. The first $24 \mathrm{~h}$ after hemorrhage show very dynamic changes in the number of neutrophils observed in the CNS. A high number of neutrophils are detected in the CNS within 10 min of the hemorrhagic event [12]. Most of these cells are localized to the microvasculature and not in the parenchyma of the brain. However, a few neutrophils do extravasate into the brain parenchyma. The number of infiltrating neutrophils within the CNS remains constant for the first $24 \mathrm{~h}$. Interestingly, this infiltration coincides with the increase in microglial activation. Our lab has shown that the activated microglia are found in close proximity to the infiltrated neutrophils at this time point [44]. We postulate the neutrophil increase/infiltration is due to signaling from blood breakdown products, inflammatory cells, or CNS vascular endothelium in response to the initial injury [44]. The inactivation or depletion of neutrophils at this time point or before the hemorrhage mitigates the development of DCI, neuronal loss, production of inflammatory cytokines in the brain, and the production of reactive oxygen species $[44,67,69]$. In addition, removal of neutrophils ameliorates hypoperfusion of the brain likely through reestablishment of vascular tone [40]. This suggests that the increased trafficking of neutrophils in CNS early after SAH 
may block the microvasculature in the brain. Therefore, early increased neutrophil localization in the brain vasculature may aggravate the ischemic milieu. It is still unclear the degree of inactivation necessary to prevent the early vascular consequences. A protocol with submaximal depletion of neutrophils from the vasculature [7] may suffice to abolish the early brain injury signs without affecting the peripheral neutrophil population. Finally, it still remains to be determined whether this first wave of neutrophil infiltration is from the original hemorrhage or recruitment from a marginated pool of neutrophils in the vicinity of the CNS or an active recruitment process mediated by the damaged/reactive blood vessels.

\section{Sub-acute Injury Period}

A second increase in brain infiltrating neutrophils has been shown at 3 days after SAH $[18,44,67,69]$. Our laboratory has demonstrated that the presence of neutrophils on day 3 after hemorrhage is critical to DND development. Reducing neutrophil recruitment and extravasation during this sub-acute period reduced proinflammatory expression, neuronal cell death, and reactive oxygen species within the CNS with no effect on brain edema [69], suggesting a continuous recruitment of these cells occurs in the CNS after SAH. The question remains as to whether this recruitment is mediated by signals released from persisting vascular dysfunction, i.e., endothelial cell injury, or activated cells from the parenchyma.

\section{Delayed and Chronic Injury Period}

Like the microglia population, very little is known about neutrophils in the chronic phase of SAH. Most research, including our own, focuses on neutrophil extravasation in the acute and sub-acute phases of the injury. Recent evidence show neutrophil infiltration into the CNS persists for many days after SAH. On day 4 after SAH, neutrophil accumulation in the brain vasculature increases microglia activation [2]. This increase may be due to factors released by neutrophils that activate the endothelium or facilitate further neutrophil extravasation into the brain. However, by day 7, the number of neutrophils in the parenchyma reduces to normal uninjured values [18]. These data raise the question as to the fate of these cells within the CNS after infiltration. Under healthy conditions, neutrophils traffic to the bone marrow, spleen, and lungs to be degraded [28]. However, during injury, resident macrophages are tasked to actively remove neutrophils from injured tissue in order to mitigate their effect on healthy tissue [28]. It would be interested to determine whether microglia in the brain actively remove infiltrating neutrophils from the brain after SAH. Finally, data showing increased microglia activation at times of increased neutrophil infiltration (acute [44], and chronic [2]),
Fig. 2 Possible cell interaction important in the development of delayed cerebral injury after SAH. Neutrophils and microglia appear to have a role in the pathophysiology that affects neuronal survival. The function of monocytes, macrophages, astrocytes, and oligodendrocyte precursor cells (OPCs) are less well studied

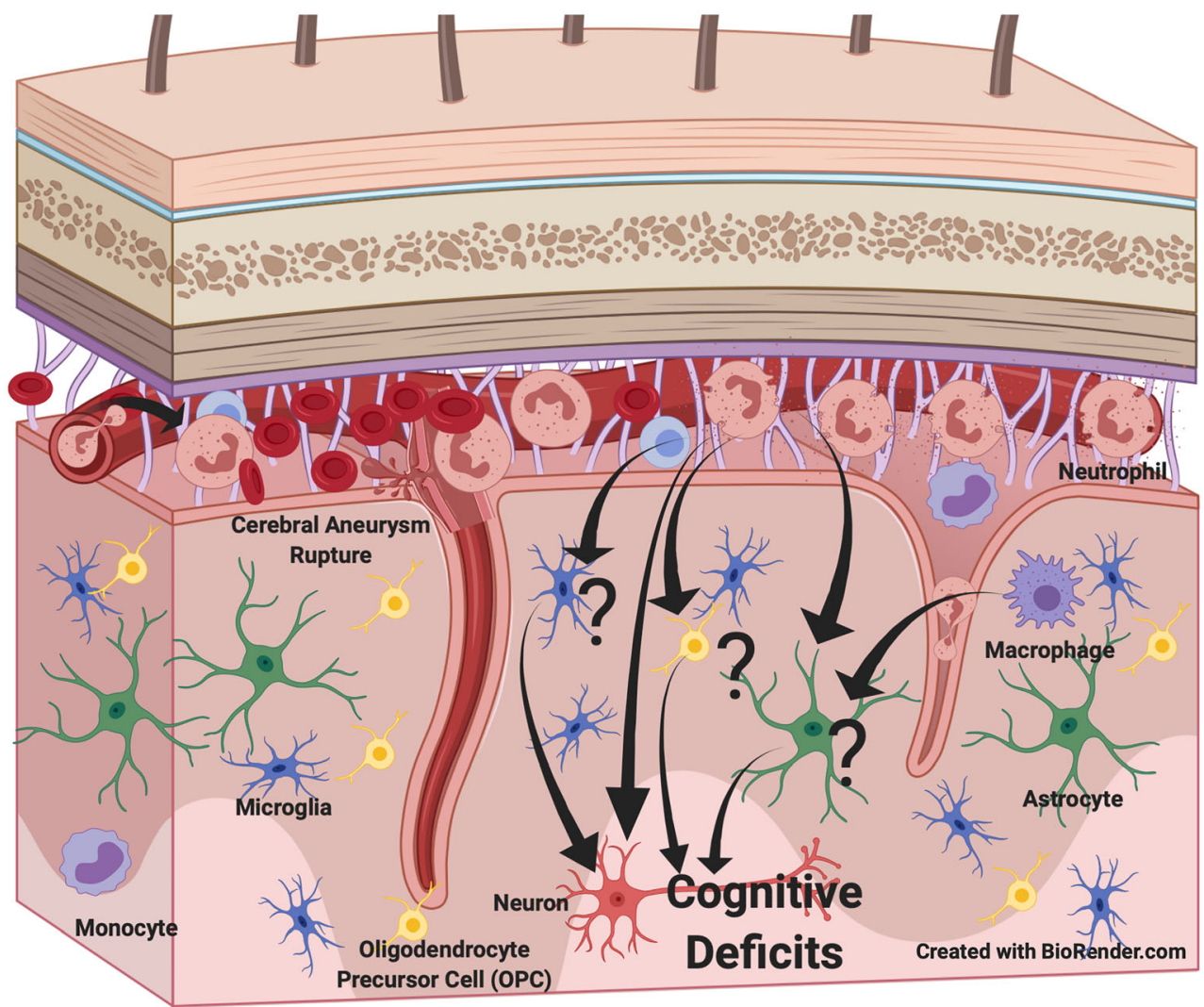


raises the question as to whether microglia activation correlated with neutrophil recruitment, or vice versa, or whether these are independent events mediated by parenchymal changes.

It is important to note that although neutrophil extravasation has been studied in $\mathrm{SAH}$, most data relate to infiltration into the parenchyma in the acute period after SAH. The exact localization of neutrophils in the CNS (vascular, parenchymal, or meningeal) in the chronic period of SAH is not known. It is curious that during this early chronic phase when microglia are re-activated, the blood-brain barrier is compromised, vasospasm recurs, and neurogenesis peaks, that so little is known about how neutrophils act. To our knowledge, our laboratory is the first to show that the chemokine, interleukin-17 (IL-17), is critical to the recruitment of neutrophils to the CNS in the chronic phase of SAH [6]. Indeed, our results demonstrate that the increased neutrophil recruitment is due to the release of IL17 by meningeal neutrophils during the chronic phase of the injury. Whether the initial recruitment is due to the activity of neutrophils from the original hemorrhagic event or the activity of other meningeal immune cells still remains to be determined. In all, further study of this time period is warranted to better elucidate the role/interaction of these immune cells with the CNS.

\section{Pathways for Inflammation: Glymphatic and Lymphatics}

SAH is characterized by the collection of blood in the subarachnoid space. A space located between the arachnoid and pial layers of the meninges. The meninges contain a host of immune cells that are proposed to act as a source of immune cells for the brain in both disease and injury [23, 39]. Furthermore, these meningeal immune cells can modulate the function of the CNS [27]. How information from inflammatory cells gets to the systemic immune system to trigger the activation of peripheral infiltrating innate immune cells is still not completely understood. Recently, lymphatic vessels have been described within the meninges that sample antigens from the CNS and process them in deep cervical lymph nodes [33]. In addition, these vessels have been implicated in the clearance of molecules from the brain [8]. Along with the description of a system for clearing molecules from the brain interstitial fluid, the glymphatic system, a picture has emerged of an organized pathway for protein clearance and presentation of antigens where interstitial fluid is moved through the glymphatic system to the cerebrospinal fluid where it is sampled by meningeal lymphatic vessels and transmitted to lymph nodes for processing and inflammatory cell recruitment [48].

A recent study of lymphatic and glymphatic system integrity after SAH demonstrates that SAH impairs the draining of the lymphatic vessels located in the meninges for up to a week after the hemorrhage [47]. It is hypothesized that this block leads to the accumulation of blood products within the perivascular space, effectively restricting the movement of molecules through the glymphatic system. Consequently, this would lead to the accumulation of toxic and inflammatory molecules within the brain parenchyma possibly leading to increased neuronal loss and cognitive deficits after SAH.

\section{Conclusion}

Long-term deficits associated with SAH have been attributed to the inflammatory state of the brain in the days to weeks after the initial hemorrhage. Although the events in the acute phase (EBI) and the delayed phase (DND) have been investigated, the state of the brain in the sub-acute and chronic phase of this injury is less well understood. Furthermore, although inflammatory modulators have been defined in the etiology of SAH, little is understood of the changes that occur in parenchymal supporting cells of the brain, the peripheral immune cells, and their contribution to the development of DND after SAH (Fig. 2). We propose that understanding both cellular and molecular changes in the brain during all phases of SAH is paramount to devising the most effective therapies to mitigate the effect of this debilitating injury, and help in the development of therapies for patients.

\section{References}

1. Altay, T., Smithason, S., Volokh, N., et al. (2009) A novel method for subarachnoid hemorrhage to induce vasospasm in mice. Journal of Neuroscience Methods, 183, 136-140. doi:https://doi.org/10. 1016/j.jneumeth.2009.06.027

2. Atangana, E., Schneider, U. C., Blecharz, K., et al. (2017) Intravascular Inflammation Triggers Intracerebral Activated Microglia and Contributes to Secondary Brain Injury After Experimental Subarachnoid Hemorrhage (eSAH). Translational Stroke Research, 8, 144-156. doi:https://doi.org/10.1007/s12975016-0485-3

3. Barz, H., Schreiber, A., \& Barz, U. (2017) Demyelinating diseases as a result of cerebral edema? Medical Hypotheses, 104, 10-14. doi: https://doi.org/10.1016/j.mehy.2017.05.010

4. Cahill, J., Calvert, J. W., Solaroglu, I., et al. (2006) Vasospasm and p53-induced apoptosis in an experimental model of subarachnoid hemorrhage. Stroke, 37, 1868-74. doi:https://doi.org/10.1161/01. STR.0000226995.27230.96

5. Chaichana, K. L., Pradilla, G., Huang, J., et al. (2010) Role of inflammation (leukocyte-endothelial cell interactions) in vasospasm after subarachnoid hemorrhage. World Neurosurgery, 73, 22-41. doi:https://doi.org/10.1016/j.surneu.2009.05.027

6. Coulibaly, A. P., Gartman, W. T., Swank, V., et al. (2019) RARRelated Orphan Receptor Gamma T (RoR $\gamma \mathrm{t})$-Related Cytokines Play a Role in Neutrophil Infiltration of the Central Nervous System After Subarachnoid Hemorrhage. Neurocritical Care doi: https://doi.org/10.1007/s12028-019-00871-9

7. Cruz Hernández, J. C., Bracko, O., Kersbergen, C. J., et al. (2019) Neutrophil adhesion in brain capillaries reduces cortical blood flow and impairs memory function in Alzheimer's disease mouse 
models. Nature Neuroscience doi:https://doi.org/10.1038/s41593018-0329-4

8. Da Mesquita, S., Fu, Z., \& Kipnis, J. (2018) The Meningeal Lymphatic System: A New Player in Neurophysiology. Neuron, 100, 375-388. doi:https://doi.org/10.1016/j.neuron.2018.09.022

9. Dodel, R., Winter, Y., Ringel, F., et al. (2010) Cost of illness in subarachnoid hemorrhage: A German Longitudinal Study. Stroke, 41, 2918-2923. doi:https://doi.org/10.1161/STROKEAHA.110. 586826

10. Fein, J. M. (1976) Brain energetics and circulatory control after subarachnoid hemorrhage. Journal of Neurosurgery, 45, 498-507. doi:https://doi.org/10.3171/jns.1976.45.5.0498

11. Fernandez-Castaneda, A., \& Gaultier, A. (2016) Adult oligodendrocyte progenitor cells - Multifaceted regulators of the CNS in health and disease. Brain, Behavior, and Immunity, 57, 1-7. doi:https://doi. org/10.1016/j.bbi.2016.01.005

12. Friedrich, V., Flores, R., Muller, A., et al. (2011) Reduction of neutrophil activity decreases early microvascular injury after subarachnoid haemorrhage. Journal of Neuroinflammation, 8, 103. doi:https://doi.org/10.1186/1742-2094-8-103

13. Friedrich, V., Flores, R., \& Sehba, F. A. (2012) Cell death starts early after subarachnoid hemorrhage. Neuroscience Letters, 512, 6 11. doi:https://doi.org/10.1016/j.neulet.2012.01.036

14. Geraghty, J. R., Davis, J. L., \& Testai, F. D. (2019) Neuroinflammation and Microvascular Dysfunction After Experimental Subarachnoid Hemorrhage: Emerging Components of Early Brain Injury Related to Outcome. Neurocritical Care, 31, 373-389. doi:https://doi.org/10.1007/s12028-019-00710-x

15. Ghaemi, A., Alizadeh, L., Babaei, S., et al. (2018) Astrocytemediated inflammation in cortical spreading depression. Cephalalgia : An International Journal of Headache, 38, 626638. doi:https://doi.org/10.1177/0333102417702132

16. Ghaemi, A., Sajadian, A., Khodaie, B., et al. (2016) Immunomodulatory Effect of Toll-Like Receptor-3 Ligand Poly I:C on Cortical Spreading Depression. Molecular Neurobiology, 53, 143-154. doi:https://doi.org/10.1007/s12035-014-8995-z

17. Grinstein, S., \& Klip, A. (1989) Calcium homeostasis and the activation of calcium channels in cells of the immune system. Bulletin of the New York Academy of Medicine, 65, 69-79. Retrieved from http://www.ncbi.nlm.nih.gov/pubmed/2557949

18. Gris, T., Laplante, P., Thebault, P., et al. (2019) Innate immunity activation in the early brain injury period following subarachnoid hemorrhage. Journal of Neuroinflammation, 16, 253. doi:https:// doi.org/10.1186/s12974-019-1629-7

19. Haage, V., Semtner, M., Vidal, R. O., et al. (2019) Comprehensive gene expression meta-analysis identifies signature genes that distinguish microglia from peripheral monocytes/macrophages in health and glioma. Acta Neuropathologica Communications, 7, 20. doi: https://doi.org/10.1186/s40478-019-0665-y

20. Hamming, A. M., Wermer, M. J. H., Umesh Rudrapatna, S., et al. (2016) Spreading depolarizations increase delayed brain injury in a rat model of subarachnoid hemorrhage. Journal of Cerebral Blood Flow and Metabolism : Official Journal of the International Society of Cerebral Blood Flow and Metabolism, 36, 1224-31. doi:https:// doi.org/10.1177/0271678X15619189

21. Hanafy, K. A. (2013) The role of microglia and the TLR4 pathway in neuronal apoptosis and vasospasm after subarachnoid hemorrhage. Journal of Neuroinflammation, 10, 83. doi:https://doi.org/ 10.1186/1742-2094-10-83

22. Hartings, J. A., York, J., Carroll, C. P., et al. (2017) Subarachnoid blood acutely induces spreading depolarizations and early cortical infarction. Brain : A Journal of Neurology, 140, 2673-2690. doi: https://doi.org/10.1093/brain/awx214

23. Herz, J., Filiano, A. J., Smith, A., et al. (2017) Myeloid Cells in the Central Nervous System. Immunity, 46, 943-956. doi:https://doi. org/10.1016/j.immuni.2017.06.007
24. Jedrzejowska-Szypułka, H., Straszak, G., Larysz-Brysz, M., et al. (2010) Interleukin-1beta plays a role in the activation of peripheral leukocytes after blood-brain barrier rupture in the course of subarachnoid hemorrhage. Current Neurovascular Research, 7, 39-48. Retrieved from http://www.ncbi.nlm.nih.gov/pubmed/20158463

25. Kassell, N. F., Sasaki, T., Colohan, A. R., et al. (1985) Cerebral vasospasm following aneurysmal subarachnoid hemorrhage. Stroke, 16, 562-72. doi:https://doi.org/10.1161/01.str.16.4.562

26. Kawabori, M., \& Yenari, M. A. (2015) The role of the microglia in acute CNS injury. Metabolic Brain Disease, 30, 381-92. doi:https:// doi.org/10.1007/s11011-014-9531-6

27. Kipnis, J. (2018) The Seventh Sense. Scientific American, 319, 28 35. doi:https://doi.org/10.1038/scientificamerican0818-28

28. Kolaczkowska, E., \& Kubes, P. (2013) Neutrophil recruitment and function in health and inflammation. Nature Reviews Immunology, 13, 159-175. doi:https://doi.org/10.1038/nri3399

29. Kooijman, E., Nijboer, C. H., van Velthoven, C. T. J., et al. (2014) Long-term functional consequences and ongoing cerebral inflammation after subarachnoid hemorrhage in the rat. PloS One, 9, e90584. doi:https://doi.org/10.1371/journal.pone.0090584

30. Lawton, M. T., \& Vates, G. E. (2017) Subarachnoid Hemorrhage. The New England Journal of Medicine, 377, 257-266. doi:https:// doi.org/10.1056/NEJMcp1605827

31. Lee, W.-D., Wang, K.-C., Tsai, Y.-F., et al. (2016) Subarachnoid Hemorrhage Promotes Proliferation, Differentiation, and Migration of Neural Stem Cells via BDNF Upregulation. PloS One, 11, e0165460. doi:https://doi.org/10.1371/journal.pone.0165460

32. Liu, F., Lu, J., Manaenko, A., et al. (2018) Mitochondria in Ischemic Stroke: New Insight and Implications. Aging and Disease, 9, 924-937. doi:https://doi.org/10.14336/AD.2017.1126

33. Louveau, A., Smirnov, I., Keyes, T. J., et al. (2015) Structural and functional features of central nervous system lymphatic vessels. Nature, 523, 337-341. doi:https://doi.org/10.1038/nature14432

34. Lucke-Wold, B. P., Logsdon, A. F., Manoranjan, B., et al. (2016) Aneurysmal subarachnoid hemorrhage and neuroinflammation: A comprehensive review. International Journal of Molecular Sciences, 17, 1-17. doi:https://doi.org/10.3390/ijms 17040497

35. Macdonald, R L, \& Weir, B. K. (1991) A review of hemoglobin and the pathogenesis of cerebral vasospasm. Stroke, 22, 971-82. doi: https://doi.org/10.1161/01.str.22.8.971

36. Macdonald, R Loch (2012) Endothelin antagonists in subarachnoid hemorrhage: what next? Critical Care (London, England), 16, 171. doi:https://doi.org/10.1186/cc11822

37. MacVicar, B. A., \& Newman, E. A. (2015) Astrocyte regulation of blood flow in the brain. Cold Spring Harbor Perspectives in Biology, 7. doi:https://doi.org/10.1101/cshperspect.a020388

38. Meguro, T., Chen, B., Lancon, J., et al. (2001) Oxyhemoglobin induces caspase-mediated cell death in cerebral endothelial cells. Journal of Neurochemistry, 77, 1128-35. doi:https://doi.org/10. 1046/j.1471-4159.2001.00313.x

39. Mrdjen, D., Pavlovic, A., Hartmann, F. J., et al. (2018) HighDimensional Single-Cell Mapping of Central Nervous System Immune Cells Reveals Distinct Myeloid Subsets in Health, Aging, and Disease. Immunity, 48, 380-395.e6. doi:https://doi. org/10.1016/j.immuni.2018.01.011

40. Neulen, A., Pantel, T., Kosterhon, M., et al. (2019) Neutrophils mediate early cerebral cortical hypoperfusion in a murine model of subarachnoid haemorrhage. Scientific Reports, 9, 8460. doi: https://doi.org/10.1038/s41598-019-44906-9

41. Nishikawa, H., \& Suzuki, H. (2018) Possible Role of Inflammation and Galectin-3 in Brain Injury after Subarachnoid Hemorrhage. Brain Sciences, 8. doi:https://doi.org/10.3390/brainsci8020030

42. Pan, L., Zhu, W., Li, Y., et al. (2014) Astrocytic Toll-like receptor 3 is associated with ischemic preconditioning-induced protection against brain ischemia in rodents. PloS One, 9, e99526. doi: https://doi.org/10.1371/journal.pone.0099526 
43. Park, S., Yamaguchi, M., Zhou, C., et al. (2004) Neurovascular protection reduces early brain injury after subarachnoid hemorrhage. Stroke, 35, 2412-2417. doi:https://doi.org/10.1161/01.STR. 0000141162.29864.e9

44. Provencio, J. Javier, Altay, T., Smithason, S., et al. (2011) Depletion of $\mathrm{Ly} 6 \mathrm{G} / \mathrm{C}+$ cells ameliorates delayed cerebral vasospasm in subarachnoid hemorrhage. Journal of Neuroimmunology, 232, 94-100. doi:https://doi.org/10.1016/j.jneuroim.2010.10.016

45. Provencio, Jose Javier, Swank, V., Lu, H., et al. (2016) Neutrophil depletion after subarachnoid hemorrhage improves memory via NMDA receptors. Brain, Behavior, and Immunity, 54, 233-242. doi:https://doi.org/10.1016/j.bbi.2016.02.007

46. Prunell, G. F., Svendgaard, N.-A., Alkass, K., et al. (2005) Delayed cell death related to acute cerebral blood flow changes following subarachnoid hemorrhage in the rat brain. Journal of Neurosurgery, 102, 1046-54. doi:https://doi.org/10.3171/jns.2005.102.6.1046

47. $\mathrm{Pu}, \mathrm{T}$., Zou, W., Feng, W., et al. (2019) Persistent Malfunction of Glymphatic and Meningeal Lymphatic Drainage in a Mouse Model of Subarachnoid Hemorrhage. Experimental Neurobiology, 28, 104-118. doi:https://doi.org/10.5607/en.2019.28.1.104

48. Raper, D., Louveau, A., \& Kipnis, J. (2016) How Do Meningeal Lymphatic Vessels Drain the CNS? Trends in Neurosciences, 39, 581-586. doi:https://doi.org/10.1016/j.tins.2016.07.001

49. Rincon, F., Rossenwasser, R. H., \& Dumont, A. (2013) The epidemiology of admissions of nontraumatic subarachnoid hemorrhage in the United States. Neurosurgery, 73, 217-22; discussion 212-3. doi:https://doi.org/10.1227/01.neu.0000430290.93304.33

50. Rinkel, G. J. E., Feigin, V. L., Algra, A., et al. (2005) Calcium antagonists for aneurysmal subarachnoid haemorrhage. The Cochrane Database of Systematic Reviews, CD000277. doi: https://doi.org/10.1002/14651858.CD000277.pub2

51. Rivero-Arias, O., Gray, A., \& Wolstenholme, J. (2010) Burden of disease and costs of aneurysmal subarachnoid haemorrhage (aSAH) in the United Kingdom. Cost Effectiveness and Resource Allocation : C/E, 8, 6. doi:https://doi.org/10.1186/1478-7547-8-6

52. Rollins, S., Perkins, E., Mandybur, G., et al. (2002) Oxyhemoglobin produces necrosis, not apoptosis, in astrocytes. Brain Research, 945, 41-9. doi:https://doi.org/10.1016/s00068993(02)02562-3

53. Sakowitz, O. W., Santos, E., Nagel, A., et al. (2013) Clusters of spreading depolarizations are associated with disturbed cerebral metabolism in patients with aneurysmal subarachnoid hemorrhage. Stroke, 44, 220-3. doi:https://doi.org/10.1161/STROKEAHA.112. 672352

54. Schallner, N., Pandit, R., LeBlanc, R., et al. (2015) Microglia regulate blood clearance in subarachnoid hemorrhage by heme oxygenase-1. Journal of Clinical Investigation, 125, 2609-2625. doi: https://doi.org/10.1172/JCI78443

55. Schneider, U C, Xu, R., \& Vajkoczy, P. (2018) Inflammatory Events Following Subarachnoid Hemorrhage (SAH). Current Neuropharmacology, 16, 1385-1395. doi:https://doi.org/10.2174/ 1570159X16666180412110919

56. Schneider, Ulf C, Davids, A.-M., Brandenburg, S., et al. (2015) Microglia inflict delayed brain injury after subarachnoid hemorrhage. Acta Neuropathologica, 130, 215-31. doi:https://doi.org/ 10.1007/s00401-015-1440-1

57. Sehba, F. A., \& Friedrich, V. (2015) Early events after aneurysmal subarachnoid hemorrhage. Acta Neurochirurgica. Supplement, 120, 23-8. doi:https://doi.org/10.1007/978-3-319-04981-6_4

58. Sehba, F. A., Pluta, R. M., \& Zhang, J. H. (2011) Metamorphosis of subarachnoid hemorrhage research: From delayed vasospasm to early brain injury. Molecular Neurobiology, 43, 27-40. doi:https:// doi.org/10.1007/s12035-010-8155-Z

59. Seo, J. H., Miyamoto, N., Hayakawa, K., et al. (2013) Oligodendrocyte precursors induce early blood-brain barrier opening after white matter injury. The Journal of Clinical Investigation, 123, 782-6. doi:https://doi.org/10.1172/JCI65863

60. Suarez, J. I. (2015) Diagnosis and management of subarachnoid haemorrhage. Continuum (Minneapolis, Minn.), 86, 25-26. doi: https://doi.org/10.1212/CON.0000000000000217

61. Sun, Q., Wu, W., Hu, Y.-C., et al. (2014) Early release of highmobility group box 1 (HMGB1) from neurons in experimental subarachnoid hemorrhage in vivo and in vitro. Journal of Neuroinflammation, 11, 106. doi:https://doi.org/10.1186/17422094-11-106

62. Tvrdik, P., Kearns, K. N., Sharifi, K. A., et al. (2019) Calcium Imaging of Microglial Network Activity in Stroke. Methods in Molecular Biology (Clifton, N.J.), 2034, 267-279. doi:https://doi. org/10.1007/978-1-4939-9658-2 19

63. Wang, Z., Shi, X.-Y., Yin, J., et al. (2012) Role of autophagy in early brain injury after experimental subarachnoid hemorrhage. Journal of Molecular Neuroscience : MN, 46, 192-202. doi: https://doi.org/10.1007/s12031-011-9575-6

64. Wei, S., Luo, C., Yu, S., et al. (2017) Erythropoietin ameliorates early brain injury after subarachnoid haemorrhage by modulating microglia polarization via the EPOR/JAK2-STAT3 pathway. Experimental Cell Research, 361, 342-352. doi:https://doi.org/10. 1016/j.yexcr.2017.11.002

65. Weir, B., Grace, M., Hansen, J., et al. (1978) Time course of vasospasm in man. Journal of Neurosurgery, 48, 173-8. doi:https://doi. org/10.3171/jns.1978.48.2.0173

66. Wilkins, A., Majed, H., Layfield, R., et al. (2003) Oligodendrocytes promote neuronal survival and axonal length by distinct intracellular mechanisms: a novel role for oligodendrocyte-derived glial cell line-derived neurotrophic factor. The Journal of Neuroscience : The Official Journal of the Society for Neuroscience, 23, 4967-74. doi: 12832519

67. Xu, H., Testai, F. D., Valyi-Nagy, T., et al. (2015) VAP-1 blockade prevents subarachnoid hemorrhage-associated cerebrovascular dilating dysfunction via repression of a neutrophil recruitment-related mechanism. Brain Research, 1603, 141-9. doi:https://doi.org/10. 1016/j.brainres.2015.01.047

68. Xu, Z., Shi, W.-H., Xu, L.-B., et al. (2019) Resident Microglia Activate before Peripheral Monocyte Infiltration and p75NTR Blockade Reduces Microglial Activation and Early Brain Injury after Subarachnoid Hemorrhage. ACS Chemical Neuroscience, 10, 412-423. doi:https://doi.org/10.1021/acschemneuro.8b00298

69. Ye, Z.-N., Wu, L.-Y., Liu, J.-P., et al. (2018) Inhibition of leukotriene B4 synthesis protects against early brain injury possibly via reducing the neutrophil-generated inflammatory response and oxidative stress after subarachnoid hemorrhage in rats. Behavioural Brain Research, 339, 19-27. doi:https://doi.org/10.1016/j.bbr. 2017.11.011

70. Yuan, S., Yu, Z., Zhang, Z., et al. (2019) RIP3 participates in early brain injury after experimental subarachnoid hemorrhage in rats by inducing necroptosis. Neurobiology of Disease, 129, 144-158. doi: https://doi.org/10.1016/j.nbd.2019.05.004

71. Zhao, X., Eyo, U. B., Murugan, M., et al. (2018) Microglial interactions with the neurovascular system in physiology and pathology. Developmental Neurobiology, 78, 604-617. doi:https://doi.org/10. 1002/dneu.22576

72. Zuo, Y., Wang, J., Enkhjargal, B., et al. (2019) Neurogenesis changes and the fate of progenitor cells after subarachnoid hemorrhage in rats. Experimental Neurology, 311, 274-284. doi:https://doi.org/10. 1016/j.expneurol.2018.10.011

Publisher's Note Springer Nature remains neutral with regard to jurisdictional claims in published maps and institutional affiliations. 